\title{
Clinical and Pathological Analyses of Borderline Changes Cases after Kidney Transplantation
}

\author{
Tomokazu Shimizu ${ }^{a, b}$ \\ aDepartment of Transplant Surgery and Urology, Toda Chuo General Hospital, Saitama, Japan; ${ }^{b}$ Department of \\ Urology, Tokyo Women's Medical University, Tokyo, Japan
}

\section{Keywords}

Acute renal rejection · Allograft kidney · Borderline changes . Kidney transplantation $\cdot$ Renal allograft biopsy

\begin{abstract}
Aim: We aimed to perform a clinicopathological analysis of cases presenting with borderline changes $(\mathrm{BC})$ after renal transplantation and discuss whether $\mathrm{BC}$ might be clinically or pathologically important. Materials and Methods: $\mathrm{BC}$ was diagnosed in 22 renal allograft biopsy specimens obtained from 20 renal transplant recipients between April 2010 and March 2019 after follow-up at the Department of Transplant Surgery, Kidney Center, Toda Chuo General Hospital. Results: $B C$ was diagnosed at a median of 500 days following transplantation. Among the 22 renal allograft biopsy specimens showing evidence of $B C$, tubulitis was observed in all specimens. Interstitial inflammation was present in $18 \mathrm{spec}-$ imens (82\%), peritubular capillaritis in $14(64 \%)$, interstitial fibrosis (ci) and tubular atrophy (ct) in 4 (18\%), and C4d deposition in the peritubular capillary was present in 6 specimens (27\%). Glomerulitis and intimal arteritis were not observed. There was no renal graft loss during the observation period, but deterioration of renal allograft function after biopsy occurred in 9 patients (45\%). Conclusions: In BC, tubulitis and interstitial inflammation were the main constitu-
\end{abstract}

karger@karger.com

(c) 2020 S. Karger AG, Basel

www.karger.com/nef

Karger" ents. Because glomerulitis was not observed in our study, we suspect that BC contributes to acute T-cell-mediated rejection. Although $B C$ did not lead to renal graft loss, renal graft function deterioration was seen in nearly half of the patients after the renal graft biopsy. We conclude that $\mathrm{BC}$ is important clinically and pathologically and needs to be monitored and treated appropriately.

(c) 2020 S. Karger AG, Basel

\section{Introduction}

Borderline changes (BCs) are defined in the Banff classification as suspicious (borderline) for acute T-cell-mediated rejection (TCMR) [1]. In the Banff 2017 classification, $\mathrm{BC}$ is defined as "foci of tubulitis (Banff $\mathrm{t}$-score $>0$ ) with minor interstitial inflammation (Banff i-score 0 or 1), or moderate-severe interstitial inflammation (i2 or i3) with mild tubulitis ( $\mathrm{t} 1$ )" [2]. The reported incidence of BC ranges from 2 to $49 \%$ and from 46 to $74 \%$ in dysfunctional graft biopsies [3-6]. At the recent Banff meeting, the elimination of BC from the Banff classification was debated [2]. In this report, we present the results of our clinical and pathological analyses of cases of $\mathrm{BC}$ after kidney transplantation and discuss whether $\mathrm{BC}$ might be important clinically and pathologically.

\footnotetext{
Tomokazu Shimizu

Department of Transplant Surgery, Toda Chuo General Hospital 1-19-3, Honcho

Toda-City 335-0023 (Japan)

houichi0114@yahoo.co.jp
} 


\section{Materials and Methods}

Between April 2010 and March 2019, BCs were diagnosed in 22 renal allograft biopsy specimens obtained from 20 renal transplant patients after follow-up at the Department of Transplant Surgery and Urology, Toda Chuo General Hospital. In this study, the data of 22 renal allograft biopsy specimens obtained from 20 patients were retrospectively reviewed from the clinical records and analyzed. The renal allograft biopsies were performed during diagnostic workup for allograft dysfunction or proteinuria (episode biopsy), or as a protocol biopsy. The renal allograft biopsy specimens were examined by light, electron, and immunofluorescence microscopy. The diagnoses were made according to the Banff 2017 classification [2]. We used the "PTCBM score" for quantifying the degree of thickening of the peritubular capillary (PTC) basement membrane evaluated by light microscopy, rather than electronmicroscopic assessment of PTC basement membrane multilayering [7]. According to the Banff 2017 classification, BC is defined as "foci of tubulitis (Banff $t$-score $>0$ ) with minor interstitial inflammation (Banff i-score 0 or 1), or moderate-severe interstitial inflammation (i2 or i3) with mild (t1) tubulitis" [2]. Even if there was an antibody for donor-specific antihuman leukocyte antigen (DSA), when the renal allograft biopsy specimen did not satisfy the histological criteria for active antibody-mediated rejection (AMR), we diagnose $\mathrm{BC}$ not as an active AMR. The patients' serum $\mathrm{Cr}$ levels (s-Cr) were compared during biopsy and after treatment or follow-up. The $20 \%$ rise or more of s-Cr was defined as "deterioration" of renal allograft function, whereas a decline in $20 \%$ or more as "improvement" and otherwise as "stable."

All patients provided informed consent for undergoing biopsies and collection of blood samples. This study was conducted with the approval of the Ethics Committee of Toda Chuo General Hospital.

\section{Results}

The background characteristics of the 20 patients with $\mathrm{BC}$ are shown in Table 1 . There were 13 male and 7 female patients, and the mean age at transplantation was 40.6 years. The mean age of the donors was 51.3 years. Nineteen patients received the transplant from living, related donors, and the remaining patient from a deceased donor. Three patients underwent pretransplant DSA. The immunosuppressive protocol mainly consisted of a 4-drug combination therapy with tacrolimus, mycophenolate mofetil, methylprednisolone, and basiliximab. Rituximab was also administered in 11 cases. As for the purpose of the biopsy, 11 cases were protocol biopsies and 9 cases were episode biopsies. Seven of the 9 episode biopsies were conducted to evaluate the effect of treatments on rejection, which had been diagnosed by previous biopsies. BC was diagnosed at a median of 500.5 days after transplantation, with a mean s-Cr level of $1.30 \mathrm{mg} / \mathrm{dL}$ at the time of biopsy.
Table 1. Clinical characteristics of patients $(n=20)$ with BCs diagnosed by biopsy

\begin{tabular}{|c|c|c|}
\hline & Mean & Min-max \\
\hline \multicolumn{3}{|l|}{ Recipient factors } \\
\hline Age at kidney transplantation, years & 40.6 & $18-66$ \\
\hline Gender $(\mathrm{M} / \mathrm{F})$ & $13 / 7$ & \\
\hline Duration of hemodialysis, months & 5.14 & $0-226$ \\
\hline \multicolumn{3}{|l|}{ Donor factors } \\
\hline Age at donation, years & 51.3 & $33-70$ \\
\hline \multicolumn{3}{|l|}{ Transplantation factors } \\
\hline Number of transplantation $(1 / 2)$ & $19 / 4$ & \\
\hline $\mathrm{ABO}(\mathrm{com} / \mathrm{InC} / \mathrm{MM})$ & $12 / 5 / 3$ & \\
\hline Living/deceased donor, $n$ & $19 / 1$ & \\
\hline HLA-AB mismatches, $n$ & 2.15 & $0-4$ \\
\hline HLA-DR mismatches, $n$ & 1.05 & $0-2$ \\
\hline Pretransplant DSA, $n(\%)$ & $3(22)$ & \\
\hline \multicolumn{3}{|l|}{ Immunosuppression, $n(\%)$} \\
\hline Tacrolimus $+\mathrm{MMF}+\mathrm{MP}$ & $20(100)$ & \\
\hline Basiliximab & $18(90)$ & \\
\hline RIT & $11(55)$ & \\
\hline Prior rejection, $n$ (\% of patients) & $7(35)$ & \\
\hline Active AMR & $1(5)$ & \\
\hline Acute TCMR & $5(25)$ & \\
\hline Active AMR + acute TCMR & $1(5)$ & \\
\hline \multicolumn{3}{|l|}{ Characteristics at time of biopsy } \\
\hline Purpose of biopsy (protocol/episode) & $11 / 9$ & \\
\hline Posttransplantation time, median, days & 500.5 & $10-3,189$ \\
\hline s-Cr, median, mg/dL & 1.30 & $0.81-2.21$ \\
\hline $\operatorname{Proteinuria}^{\star}(\geqq 1$, dipstic) (yes/trace/no) & $4 / 5 / 13$ & \\
\hline
\end{tabular}

Com, $\mathrm{ABO}$ compatible; $\mathrm{InC}, \mathrm{ABO}$ in compatible; $\mathrm{MM}, \mathrm{ABO}$ minor mismatch; MMF, mycophenolate mofetil; MP, methylprednisolone; AMR, antibody-mediated rejection; TCMR, T-cell-mediated rejection; HLA, human leukocyte antigen; DSA, donor-specific HLA antibody; $\mathrm{BC}$, borderline change; s-Cr, serum creatinine; RIT, rituximab. * Proteinuria at time of biopsy, which number is per biopsy time.

The histopathological findings of the 22 renal allograft biopsy specimens with $\mathrm{BC}$ are shown in Table 2. Among the 22 specimens showing evidence of $\mathrm{BC}$, tubulitis was seen in all specimens; the tubulitis was mild (Banff $t$ ) in 17 , moderate ( $\mathrm{t} 2$ ) in 2 , and severe in 3 . Interstitial inflammation was present in 18 specimens (82\%), mild (i1) in 16 , moderate (i2) in 2 specimens, and severe (i3) in 0. Peritubular capillaritis was present in 14 specimens (64\%), ci and ct in 4 specimens (18\%), and C4d deposition in the PTC in 6 specimens (27\%). Glomerulitis and intimal arteritis were not present. Arteriolar hyaline thickening was observed in 10 specimens (45\%) and calcineurin inhibitor arteriolopathy in 1 specimen (5\%).

The relations of the Banff $\mathrm{i}$-score and Banff $\mathrm{t}$-score in $\mathrm{BC}$ are illustrated in Table 3. As for the combination of
Shimizu 
Table 2. The histopathology of 22 allograft biopsies with BCs

\begin{tabular}{|c|c|c|}
\hline Banff score & $\begin{array}{l}\text { Number, } \\
n=22\end{array}$ & $\begin{array}{l}\text { Ratio of Banff } \\
\text { score } \geq 1(\%)\end{array}$ \\
\hline \multicolumn{3}{|l|}{$\mathrm{t}$} \\
\hline $\mathrm{t} 1: \mathrm{t} 2: \mathrm{t} 3$ & $17: 2: 3$ & 100 \\
\hline i1:i2:i3 & $16: 2: 0$ & 82 \\
\hline \multicolumn{3}{|l|}{ ptc } \\
\hline ptc1:ptc2:ptc3 & $12: 1: 1$ & 64 \\
\hline \multicolumn{3}{|l|}{ g } \\
\hline g1:g2:g3 & $0: 0: 0$ & 0 \\
\hline $\mathrm{v}$ & & \\
\hline \multirow{2}{*}{\multicolumn{3}{|c|}{$\begin{array}{l}\text { v1:v2:v3 } \\
\text { C4d deposition in PTC }(\mathrm{C} 4 \mathrm{~d})\end{array}$}} \\
\hline & & \\
\hline C4d1:C4d2:C4d3 & $2: 3: 1$ & 27 \\
\hline \multicolumn{3}{|l|}{$\mathrm{ci}$} \\
\hline \multicolumn{3}{|l|}{ ct } \\
\hline ct1:ct2:ct3 & $2: 2: 0$ & 18 \\
\hline \multicolumn{3}{|l|}{$\mathrm{cg}$} \\
\hline $\operatorname{cg} 1: \operatorname{cg} 2: \operatorname{cg} 3$ & $1: 0: 0$ & 5 \\
\hline \multicolumn{3}{|l|}{ ptcbm* } \\
\hline ptcbm1:ptcbm2 & $3: 0$ & 14 \\
\hline \multicolumn{3}{|l|}{$\mathrm{cV}$} \\
\hline cv1:cv2:cv3 & 0:0:0 & 0 \\
\hline \multicolumn{3}{|l|}{$\mathrm{ah}$} \\
\hline $\begin{array}{l}\text { ah1:ah2:ah3 } \\
\text { aah }\end{array}$ & $7: 2: 1$ & 45 \\
\hline aah1:aah2:aah3 & $0: 1: 0$ & 5 \\
\hline
\end{tabular}

PTC, peritubular capillary; PTCBMML, peritubular capillary basement membrane multilayering; $\mathrm{BC}$, borderline change; $\mathrm{t}, \mathrm{tu}-$ bulitis; i, interstitial inflammation; g, glomerulitis; v, intimal arteritis; ah, arteriolar hyaline thickening; aah, calcineurin inhibitor arteriolopathy; $\mathrm{cv}$, arterial fibrous intimal thickening; cg, glomerular double contours; ct, tubular atrophy; ci, interstitial fibrosis; ptcbm, PTCBMML. * ptcbm score is advocated by Aita et al. [7] which is used instead of PTCBMML.

Banff $\mathrm{t}$-score and Banff $\mathrm{i}$-score in this BC series, ilt1 was most common, being seen in 14 specimens, and $\mathrm{i} 2 \mathrm{t} 1$ and i0t3 were seen in 2 specimens each.

Treatment for BC was administered in 19 cases, with steroid pulse therapy performed in 17 cases, and the medication rituximab or intravenous high-dosage immune globulin therapy was administered in 1 case each. In 3 cases, treatment was not performed because treatment was thought to contribute to acute TCMR, which had been diagnosed by previous biopsies.

The prognosis of the patients with BCs is shown in Table 4. There was no renal graft loss or patient death during the observation period, but deterioration of renal
Table 3. Relations of Banff i-score and Banff t-score in BCs $(n=$ 22)

\begin{tabular}{lrrllc}
\hline & i0 & i1 & i2 & i3 & Total \\
\hline t1 & 1 & 14 & 2 & 0 & 17 \\
t2 & 1 & 1 & $0^{*}$ & $0^{*}$ & 2 \\
t3 & 2 & 1 & $0^{*}$ & $0^{*}$ & 3 \\
\hline Total & 4 & 16 & 2 & 0 & 22 \\
\hline
\end{tabular}

$\mathrm{BC}$, borderline change. ${ }^{*} \mathrm{i} 2$ and/or i 3 with $\mathrm{t} 2$ and/or $\mathrm{t} 3$ are defined as acute T-cell-mediated rejection, according to the Banff 2017 classification.

Table 4. Prognosis of the patients with BCs $(n=20)$

\begin{tabular}{|c|c|}
\hline Graft loss & 0 cases $(0 \%)$ \\
\hline Functioning graft & 20 cases $(100 \%)$ \\
\hline Deterioration of renal allografts' function* & 9 cases $(45 \%)$ \\
\hline Stable of renal allografts' function* & 5 cases $(25 \%)$ \\
\hline Improvement of renal allografts' function* & 6 cases $(30 \%)$ \\
\hline Death & 0 cases $(0 \%)$ \\
\hline Total & 20 cases $(100 \%)$ \\
\hline
\end{tabular}

allograft function after BC diagnosis occurred in 9 patients (45\%).

The pathological course after BC biopsy is demonstrated in Table 5. Sixteen patients received follow-up allograft biopsies after BC diagnosis with an average of 233.8 days. Resolutions of BC were proved by later allograft biopsies in 4 cases. Progression to active AMR was seen in 4 patients, and 2 of these 4 patients progressed to chronic active AMR. Progression to acute TCMR was seen in 1 patient and chronic active AMR in 1 case.

The status of circulating DSA in BC is shown in Table 6. Three patients underwent pretransplant DSA. Of the total of 14 blood samples obtained at the time of the renal allograft biopsy and after the biopsy, DSA was detected in 9 samples (40\%) with DSA class I alone in 2 samples (10\%); DSA class II alone in 6 samples (30\%); and both DSA class I and II in 1 sample (5\%). Of the 9 samples with DSA, 6 samples (30\%) showed de novo DSA, including DSA class I alone in 1 sample (5\%), class II alone in 4 samples (20\%), and both class I and class II in 1 sample (5\%). 
Table 5. Pathological course after the biopsy of BCs

\begin{tabular}{lc}
\hline No biopsy & $\mathbf{4}$ cases $(\mathbf{2 0 \%})$ \\
Follow-up biopsy & $\mathbf{1 6}$ cases $\mathbf{8 0 \% )}$ \\
$\quad$ Resolution of BCs & $\mathbf{1 0}$ cases $(\mathbf{5 0 \%})$ \\
$\quad$ Progression to rejection & $\mathbf{6}$ cases $(\mathbf{3 0 \%})$ \\
$\quad$ Progression to active AMR & $4^{*}$ cases $(20 \%)$ \\
$\quad$ Progression to acute TCMR & 1 cases $(5 \%)$ \\
$\quad$ Progression to chronic active AMR & 1 cases $(5 \%)$ \\
\hline Total & $\mathbf{2 0}$ cases $(\mathbf{1 0 0 \% )}$ \\
\hline
\end{tabular}

AMR, antibody-mediated rejection; TCMR, T-cell-mediated rejection; $\mathrm{BC}$, borderline change. ${ }^{*}$ Two of 4 were moreover progressive to chronic AMR, later.

Table 6. The status of circulating donor-specific HLA antibodies in BCs

\begin{tabular}{ll}
\hline & $N($ total 20) (\%) \\
\hline Pretransplant DSA & \\
Negative & $17(85)$ \\
Positive & $3(15)$ \\
DSA class I & $1(5)$ \\
DSA class II & $2(10)$ \\
DSA measurement & $14(70)$ \\
DSA & \\
Negative & $6(30)$ \\
Positive & $9(40)$ \\
DSA class I & $2(10)$ \\
DSA class II & $6(30)$ \\
DSA class I and class II & $1(5)$ \\
de novo DSA & $6(30)$ \\
Positive & $1(5)$ \\
DSA class I & $4(20)$ \\
DSA class II & $1(5)$ \\
DSA class I and class II &
\end{tabular}

HLA, human leukocyte antigen; DSA, donor-specific antiHLA antibody; BC, borderline change. * Blood samples obtained at the time of the renal allograft biopsy and after the biopsy.

\section{Discussion}

In this study, tubulitis was seen in all BSs, interstitial inflammation was present in $80 \%$ of BS cases, often accompanied by peritubular capillaritis $(64 \%)$, but glomerulitis and intimal arteritis were not present. Therefore, our cases might have been TCMR not AMR.

This study by Masin-Spasovska et al. [8] reported, using protocol biopsies, $\mathrm{BC}$ might occur in patients with stable graft function. In our study, $11 \mathrm{BC}$ cases were diag- nosed by protocol biopsies, which had stable renal allograft function. The biological process of rejection is a gradual process that culminates in full-blown rejection [9]. If a biopsy is performed very early during the course, the pathological lesions will not have reached the threshold for the diagnosis of rejection [9]. We suggest that in our protocol biopsy cases, we performed biopsies before shifting to complete acute TCMR. From this theory, in these cases, BC might be an early form of acute TCMR. Seven of 9 of the episode biopsies were conducted by evaluating the effect of treatments on rejection, which had been diagnosed by previous biopsies. In these cases, BC might be a process in the middle of the resolution of the rejection.

As for treatment, Min et al. [10] reported that no treatment for BC at the early protocol biopsy was identified as a risk factor for aggravating chronic injuries in the multivariate analysis and suggested that subclinical BC should be treated with steroid pulse therapy to prevent chronic allograft injury. They divided BC cases, who underwent protocol biopsies 10 days and 1 year after transplantation, into 2 groups: the treatment group with BC following steroid pulse therapy and the nontreatment group [10]. The nontreatment group had more prominent chronic scores, including the Banff Chronicity Score, which was calculated as the sum of scores for the individual histological markers for chronicity: ci (Banff ci-score), ct, vascular fibrous intimal thickening, and chronic glomerulopathy. They concluded that subclinical BC could be a risk factor for chronic allograft injury and should be considered for antirejection therapy [10]. Roberts et al. [11] reported that subclinical BC detected by protocol biopsies at day 28 was not an adverse prognostic factor for long-term outcome in terms of renal allograft function. Meehan et al. [12] showed that s-Cr increases $>110 \%$ of baseline and BC progressed to acute rejection in only $28 \%$ of untreated $\mathrm{BC}$; thus, mandatory treatment was not recommended. The treatment for our BC cases, mainly SP, was performed in 19 cases (86\%), but the resolution of $\mathrm{BC}$ was proved by later allograft biopsies in only 4 cases (21\%). From these data, we believe that treatment is necessary.

Although BC did not lead to renal graft loss, renal graft function deterioration was seen in $45 \%$ of cases following BC diagnosis. Nankivell et al. [13] reported that BC increased the rates of acute organ dysfunction. They described that renal functional recovery was incomplete in $46.3 \%$ of cases 1 month after BC. Some reports indicate the failed functional recovery after BC occurred is 25$58 \%[5,14]$. 
The progression to active AMR by follow-up biopsy was seen in 4 patients (20\%); moreover, 2 of these 4 patients progressed to chronic active AMR. Progression to acute TCMR was seen in 1 case (5\%) and chronic active AMR in 1 case (5\%). A third of the cases progressed to active AMR or acute TCMR. Nankivell et al. [13] reported that acute rejection episodes reoccurred in $50 \%$ of patients after BC, which was detected by protocol biopsy. Acute rejection episodes reoccurred in $32.6 \%$ of patients after protocol biopsy, despite antirejection treatment in $83.3 \%$ of patients.

In our study, de novo DSA appeared in $30 \%$ of cases, and 25\% shifted to AMR. Nankivell et al. [13] also noted that $\mathrm{BC}$ increased the rates of DSA generation with subsequent AMR pathology. They described that moderate DSA (MFI > 3,000) was more prevalent in BC cases than in acute TCMR cases or normal cases [13]. The 3-, 12-, and 36-month incidences of any de novo DSA for BC were $16.2,31.5$, and $56.7 \%$, respectively [13]. BC might induce the generation of de novo DSA and progress to AMR. A humoral immune response is thought to be activated by a cell-mediated immunological reaction. The onset of $\mathrm{BC}$ might be the cause of the immune stimulation, and it might be necessary to be careful clinically and pathologically.

This study has some limitations. First, in this study, BC cases included both the protocol biopsy group and the episodic biopsy group, which was aimed to confirm the graft status after rejection treatment. These 2 biopsy groups might have different characteristics. Next, this study was retrospective in nature, with a small sample size and shorter follow-up.
In conclusion, as for $\mathrm{BC}$, tubulitis and interstitial inflammation were the main constituents, often accompanied by peritubular capillaritis. Because glomerulitis was not found in our study, we agree that BC might be suspicious for acute TCMR. Although BC did not lead to renal graft loss, renal graft function deterioration was seen in nearly half of the cases after the renal graft biopsy, despite antirejection therapy. In addition, de novo DSA appeared in $30 \%$ of cases, and $25 \%$ shifted to antibody-related rejection. We conclude that $\mathrm{BC}$ is important clinically and pathologically, and it is necessary to treat it appropriately and monitor it following treatment. The prognosis of grafts exhibiting $\mathrm{BC}$ was not good even under the present immunosuppressive protocol and antirejection therapy.

\section{Acknowledgements}

We thank Shigeru Horita, Hideki Nakayama, and Mayuko Ohono (Division of Pathology, Kidney Center, Tokyo Women's Medical University) for their technical support.

\section{Statement of Ethics}

This research was conducted ethically in accordance with the World Medical Association Declaration of Helsinki. All patients provided informed consent for undergoing biopsies and collection of blood samples. This study was conducted with the approval of the Ethics Committee of Toda Chuo General Hospital.

\section{Conflict of Interest Statement}

The author has no conflicts of interest to declare.

\section{References}

1 Solez K, Axelsen RA, Benediktsson H, Burdick JF, Cohen AH, Colvin RB, et al. International standardization of criteria for the histologic diagnosis of renal allograft rejection: the Banff working classification of kidney transplant pathology. Kidney Int. 1993 Aug;44(2):41122.

2 Hass M, Loupy A, Lefaucheur C, Roufosse C, Glotz D, Seron D, et al. The Banff 2017 meeting report: revised diagnostic criterias for chronic active $\mathrm{T}$ cell-mediated rejection, antibody-mediated rejection, and prospects for integrative endpoints for next-generation clinical trials. Am J Transplant. 2018 Feb; 18(2):293-307.
3 Gaber LW, Moore LW, Gaber AO, First MR, Guttmann RD, Pouletty P, et al. A multicenter report on ultilization of Banff grading in acute renal rejection: the 1995 efficacy endpoints conference. Transplant Proc. 1997 Feb-Mar; 29(1-2):184-5.

4 Solez K, Hansen HE, Kornerup HJ, Madsen S, Sørensen AW, Pedersen EB, et al. Clinical validation and reproducibility of the Banff schema for renal allograft pathology. Transplant Proc. 1995 Feb;27(1):1009-11.

5 Schweitzer EJ, Drachenberg CB, Anderson L, Papadimetriou JC, Kuo PC, Johnson LB, et al. Significance of the Banff borderline biopsy. Am J Kidney Dis. 1996 Oct;28(4):585-8.
6 Meehan SM, Siegel CT, Aronson AJ, Bartosh SM, Thistlethwaite JR, Woodle ES, et al. The relationship of untreated borderline infiltrates by the Banff criteria to acute rejection in renal allograft biopsies. J Am Soc Nephrol. 1999 Aug;10(8):1806-14.

7 Aita K, Yamaguchi Y, Horita S, Ohno M, Tanabe K, Fuchinoue S, et al. Thickening of the peritubular capillary basement membrane is a useful diagnostic marker of chronic rejection in renal allografts. Am J Transplant. 2007 Apr; 7(4):923-9.

8 Masin-Spasovska J, Spasovski G, Dzikova S, Petrusevska G, Lekovski L, Ivanovski N, et al. Do we have to treat subclinical rejections in early protocol renal allograft biopsies? Transplant Proc. 2007 Oct;39(8):2550-3. 
9 Mubarak M, Shakeel S, Abbas K, Aziz T, Zafar MN, Naqvi SA, et al. Borderline changes on dysfunctional renal allograft biopsies: clinical relevance in a living related renal transplant setting. Exp Clin Transplant. 2017 Feb; 15(Suppl 1):24-7.

10 Min SI, Park YS, Ahn S, Park T, Park DD, Kim $\mathrm{SM}$, et al. Chronic allograft injury by subclinical borderline change: evidence from serial protocol biopsies in kidney transplantation. J Korean Surg Soc. 2012 Dec;83(6):343-51.
11 Roberts IS, Reddy S, Russell C, Davies DR, Friend PJ, Handa AI, et al. Subclinical rejection and borderline changes in early protocol biopsy specimens after renal transplantation. Transplantation. 2004 Apr;77(8):1194-8.

12 Meehan SM, Siegel CT, Aronson AJ, Bartosh SM, Thistlethwaite JR, Woodle ES, et al. The relationship of untreated borderline infiltrates by the Banff criteria to acute rejection in renal allograft biopsies. J Am Soc Nephrol. 1999 Aug;10(8):1806-14.
13 Nankivell BJ, Agrawal N, Sharma A, Taverniti A, P’Ng CH, Shingde M, et al. The clinical and pathological significance of borderline $\mathrm{T}$ cell-mediated rejection. Am J Transplant. 2019 May;19(5):1452-63.

14 Saad R, Gritsch HA, Shapiro R, Jordan M, Vivas $C$, Scantlebury V, et al. Clinical significance of renal allograft biopsies with "borderline changes," as defined in the Banff schema. Transplantation. 1997;64(7):992. 